
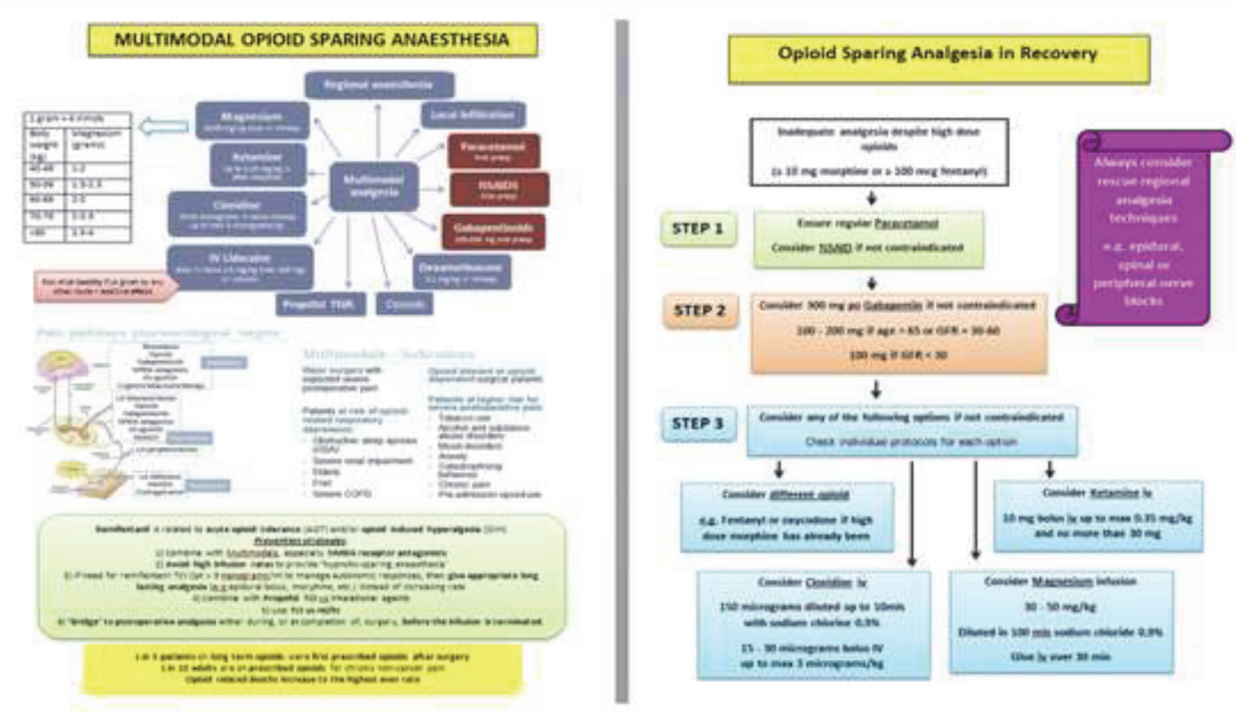

Abstract 228 Figure 1

Abstract 228 Table 1 Opioid use in theatres and recovery during the first two weeks of November 2019. IVME=IV morphine equivalents, $n=$ number of patients, median=median opioid use, PACU=post anaesthesia care unit

\begin{tabular}{|c|c|c|c|}
\hline BSUH AREA & $\begin{array}{c}\text { IVME } \\
\text { (mg) }\end{array}$ & $\mathbf{n}$ & Median (min-max) (mg) \\
\hline $\begin{array}{c}\text { Theatre 1 } \\
\text { (orthopaedic trauma) }\end{array}$ & 555.5 & 30 & $20(2.5-38)$ \\
\hline $\begin{array}{c}\text { Theatre 2 } \\
\text { (complex ENT) }\end{array}$ & 225.5 & 16 & $10(5-30)$ \\
\hline $\begin{array}{c}\text { Theatre 4 } \\
\text { (Emergencies) }\end{array}$ & 806.5 & 43 & $20(5-40)$ \\
\hline $\begin{array}{c}\text { Theatre 5 } \\
\text { (Major gynaccology and gencral } \\
\text { oncology surgery) }\end{array}$ & 260.5 & 16 & $12.5(5-30)$ \\
\hline PACU & 870 & 85 & $\mathbf{8 ( 2 - 4 5 . 4 )}$ \\
\hline
\end{tabular}

plethora of non-opioid adjuncts which may facilitate opioidfree or opioid-light anaesthesia and analgesia. Further work is required to investigate if anaesthesia and recovery opioid sparing protocols can improve patient outcomes.

\title{
229 INSTITUTIONAL ENHANCED RECOVERY PROGRAM QUALITY PAIN AUDIT
}

P-J Windal*, S Coppens. UZ Leuven, Leuven, Belgium

10.1136/rapm-2021-ESRA.229

Background and Aims Pain remains a major complaint in the immediate post-operative phase. Despite enhanced recovery protocols (ERP) and procedure specific pain therapy up to $80 \%$ of patients experience significant uncontrolled pain. Aim of this quality control pain audit was assessing pain intensity in institutional developed multimodal perioperative care pathways. Analyses of efficacy locoregional analgesia, management and protocol adherence. Determine possible improvements of guidelines and procedures

Methods Prospective data were collected on 118 patients undergoing six types of surgery. Pain scores were evaluated on

postoperative care unit (PACU) and first five days following surgery. Primary outcome was worst recorded pain score. Secondary outcomes included median pain on ward, incidence of postoperative nausea and vomiting, time to first mobilization. Following procedures were included: minimal invasive cardiac surgery, video assisted thoracic surgery, esophageal resection, total knee arthroplasty, laparoscopic colorectal surgery and robotic prostatectomy.

Results Numeric Rating Scale higher then three was observed in $89 \%$ of study population. At PACU esophageal resection showed worst pain scores despite neuraxial analgesia (Median: 7, IQR 5-8). Total knee arthoplasty resulted in worst pain scores on ward following surgery (median: 7, IQR 5-8). Disappointingly only $25 \%$ of patients received pain medication following institutional ERP guidelines. Current ERP-guideline are similar to ERAS guidelines found in literature.

Conclusions Multimodal pain therapy should become standard of care. Constant evaluation of pain guidelines are necessary. Audits and evaluation of protocol adherence next to clear guidelines and managing patient information, expectations are pivotal.

\section{CLONIDINE AS AN ADJUNCT TO LEVOBUPIVACAINE FOR PUDENDAL BLOCK DURING PROCTOLOGICAL SURGERY: A PROSPECTIVE, RANDOMISED, PILOT STUDY}

M Gambassi*, M Suball, M Bortes, P Kapessidou. CHU Saint Pierre, Brussels, Belgium

10.1136/rapm-2021-ESRA.230

Background and Aims Proctological surgery is associated with high-score postoperative pain (VAS $>5$ ) [1].

Pudendal block reduces pain and allows a faster return to normal activity [2].

Clonidine has been suggested to improve the quality and duration of peripheral blocks [3 4 ].

This study investigates whether adding clonidine to levobupivacaine in proctological surgery reduces postoperative consumption of analgesics.

Methods After local ethics committee approval and signed informed consent, adults ASA I/II, scheduled for proctologic 